\title{
A meta-analysis of technical efficiency in aquaculture
}

\begin{abstract}
We compared stochastic frontier analysis (SFA) and data envelopment analysis (DEA) methods to calculate mean technical efficiency (MTE) of global aquaculture. A total of 36 published articles on technical efficiency of 55 aquaculture operations were reviewed. SFA yielded lower MTE estimates than DEA. MTE for Asia, Africa, Europe, and the USA was estimated to be $0.64,0.71,0.80$, and 0.73 , respectively, indicating considerable scope for increasing output of aquaculture with the currently available array of inputs and existing technology.
\end{abstract}

Keyword: Stochastic frontier analysis; Data envelopment analysis; Cobb-Douglass;

Translog function form; Meta-analysis 